Draft Version November 1, 2021

Preprint typeset using $\mathrm{LATE}_{\mathrm{E}} \mathrm{X}$ style emulateapj v. 12/16/11

\title{
DANCING TWINS: STELLAR HIERARCHIES THAT FORMED SEQUENTIALLY?
}

\author{
ANDREI TOKOVININ \\ Cerro Tololo Inter-American Observatory, Casilla 603, La Serena, Chile \\ Draft version November 1, 2021
}

\begin{abstract}
This paper attracts attention to the class of resolved triple stars with moderate ratios of inner and outer periods (possibly in a mean motion resonance) and nearly circular, mutually aligned orbits. Moreover, stars in the inner pair are twins with almost identical masses, while the mass sum of the inner pair is comparable to the mass of the outer component. Such systems could be formed either sequentially (inside-out) by disk fragmentation with subsequent accretion and migration or by a cascade hierarchical fragmentation of a rotating cloud. Orbits of the outer and inner subsystems are computed or updated in four such hierarchies: LHS 1070 (GJ 2005, periods 77.6 and 17.25 years), HIP 9497 (80 and 14.4 years), HIP 25240 (1200 and 47.0 years), and HIP 78842 (131 and 10.5 years).

Subject headings: binaries:visual; binaries:general
\end{abstract}

\section{INTRODUCTION}

Interest in the architecture and dynamics of hierarchical stellar systems is stimulated by the desire to better understand their origin. Low-mass hierarchies with outer separations less than $\sim 50$ au show a tendency of orbit alignment (Tokovinin 2017), resembling in this respect multi-planet systems (Fabrycky et al. 2014), although they differ from planets in other properties. This suggests that such hierarchies could have formed and evolved in a viscous accretion disk. As the gas from the circumbinary disc is preferentially accreted by the secondary component, binaries evolve toward equal masses (twins), while their orbits shrink in size (Artymowicz \& Lubow 2001). This scenario is naturally extended to explain nearly co-planar compact hierarchies: while the inner binary shrinks, another companion can be formed by fragmentation of the circumbinary disk, possibly destabilized by an accretion burst. The newly formed outer companion overtakes the accretion and grows until its mass equals the combined mass of the inner pair. Thus, formation of double twins, where both outer and inner mass ratios are close to one, is a natural outcome of the sequential inside-out assembly of hierarchies. The triple protostellar system discovered by Tobin et al. (2016) is an excellent illustration of this scenario. Its outer component $\mathrm{C}$ is the least massive one, yet it accretes most of the gas from the circumbinary disk and will eventually catch up in mass with the inner pair A,B, unless the gas supply is exhausted or dispersed earlier or its inward migration destabilizes the system dynamically.

However, the sequential inside-out formation, depicted schematically in Figure 1(left), is not the only possibility. During collapse of pre-stellar cores, the increasing density and decreasing Jeans mass promote cascade fragmentation, starting at the largest scales and proceeding to subfragmentation into smaller parcels (Norman \& Wilson 1978; Guszeinov et al. 2017). If the mass is evenly divided between the fragments, cascade fragmentation will produce a $2+2$ quadruple system with four nearly equal components, or a double-twin triple system if only one

atokovinin@ctio.noao.edu
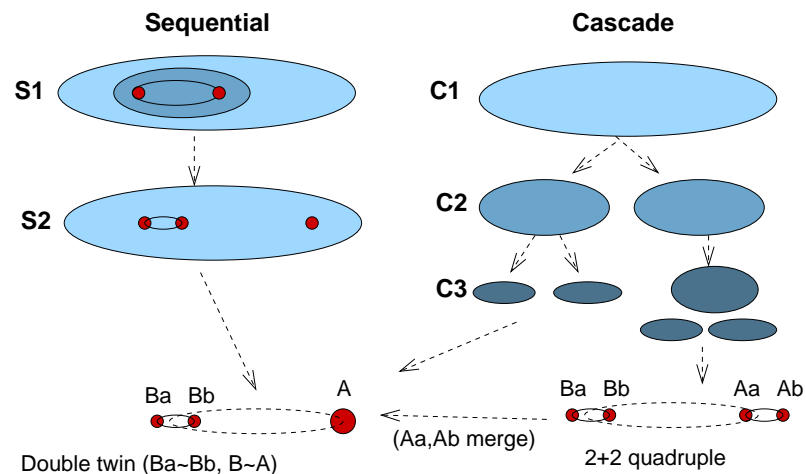

FIG. 1. - Two scenaria leading to the formation of double-twin hierarchies with approximately aligned orbits. In the sequential, inside-out formation (left), the inner binary forms first (S1), grows in mass and migrates, until the outer companion is formed (S2). The companion A can grow to the point when its mass equals the total mass of the inner binary $\mathrm{Ba}, \mathrm{Bb}$, thus producing a double twin. In the cascade (hierarchical) fragmentation scenario (right), the rotating cloud fragments into approximately equal parts (C2), and then one or both fragments further split, forming one or two inner pairs (C3). A quadruple system (Ba,Bb and $\mathrm{Aa}, \mathrm{Ab}$ ) can later become a double twin if one of its inner pairs merges.

of the outer parcels fragments or if one of the inner pairs merges during subsequent evolution (Figure1, right). In this scenario, masses of the emerging stars are set by the masses of the fragments, while the size of the outer orbit is defined by the angular momentum of the parent core.

Both formation scenarios probably occur. Massive $2+2$ quadruple systems of $\varepsilon$ Lyr type with wide outer orbits (Tokovinn 2008) match the expected outcome of cascade fragmentation. On the other hand, compact hierarchies composed of low-mass stars are more likely to be formed by disc fragmentation sequentially, inside-out. Inward migration of the outer companion can disrupt the triple system dynamically. However, before this happens, the interacting outer and inner orbits can be trapped in a mean motion resonance (MMR), as often occurs in multiplanet systems (e.g. Lee et al. 2013). Orbital motions in such hierarchies resemble a complex dance.

Detailed observational characterization of masses and orbits in a large number of hierarchies will help to decide which formation scenario dominates and will chal- 
TABLE 1

OVERVIEW OF MULTIPLE SYSTEMS

\begin{tabular}{ccclccccc}
\hline \hline WDS & $\begin{array}{c}\text { HIP } \\
\pi(\mathrm{mas})\end{array}$ & $\begin{array}{c}V(\mathrm{mag}) \\
\text { Sp.type }\end{array}$ & $\begin{array}{l}\text { Outer Name } \\
\text { Inner Name }\end{array}$ & $\begin{array}{c}P \\
(\mathrm{yr})\end{array}$ & $\begin{array}{c}a \\
\left({ }^{\prime \prime}\right)\end{array}$ & $\begin{array}{c}M_{1} \\
\left(\mathcal{M}_{\odot}\right)\end{array}$ & $\begin{array}{c}M_{2} \\
\left(\mathcal{M}_{\odot}\right)\end{array}$ & $\begin{array}{c}\Phi\left(^{\circ}\right) \\
P_{2} / P_{1}\end{array}$ \\
\hline \multirow{2}{*}{$00247-2653$} & $\ldots$ & 15.4 & LEI 1 A,BC & 77.6 & 1.528 & 0.12 & $0.15 \mathrm{~s}$ & 1,125 \\
& 129.5 & M7V & LEI 1 B,C & 17.25 & 0.460 & 0.077 & 0.070 & $4.50 \pm 0.03$ \\
$02022-2402$ & 9497 & 7.88 & HDS 272 A,B & 80 & 0.414 & 1.44 & $1.22 \mathrm{~s}$ & 27,27 \\
& 16.09 & F6V & TOK 41 Ba,Bb & 14.4 & 0.102 & 0.64 & 0.58 & $5.6 \pm 0.7$ \\
$05239-0052$ & 25240 & 6.11 & WNC 2 A,BC & 1200 & 3.716 & $2.73 \mathrm{~s}$ & $2.62 \mathrm{~s}$ & 17,166 \\
& 18.75 & F7V & A 847 B,C & 47 & 0.337 & 1.07 & 1.55 & $\sim 25$ \\
$16057-3252$ & 78842 & 8.34 & SEE 264 A,B & 131 & 0.807 & 0.96 & $1.50 \mathrm{~s}$ & 14,65 \\
& 24.70 & K0V & WSI 84 Ba,Bb & 10.5 & 0.128 & 0.75 & 0.75 & $12.6 \pm 0.2$ \\
\hline
\end{tabular}

Note. - Explanation of columns: (1) WDS code (Mason et al. 2001); (2) Hipparcos number and trigonometric parallax from various sources; (3) combined visual magnitude and spectral type from Simbad; (4) discoverer codes; (5) orbital period; (6) semimajor axis; (7) mass of the primary component; (8) mass of the secondary component (s means mass sum); (9) two values of mutual inclination $\Phi$ and the period ratio.

lenge the theory to explain their architecture. In this paper, the observed motions in four resolved hierarchical systems composed of low-mass stars are modeled by the inner and outer instantaneous Keplerian orbits. Relative orbit orientations, period ratios, and masses of the components are deduced, thus enriching the still scarce observational data on hierarchical multiple systems and contributing to their statistics, like the previous efforts in this direction (Tokovinin et al. 2015; Tokovinin \& Latham 2017). However, the hierarchies studied here are wider and slower and do not have matching radial velocity $(\mathrm{RV})$ data; their orbits are based only on resolved measurements.

In 3-body systems, the osculating orbits evolve with time. In many cases, this evolution is too slow to be detectable (e.g. Heintz 1996), otherwise a more complete dynamical analysis should be made, like in (Xu et al. 2015). Such analysis is beyond the scope of this paper.

The four multiple systems studied here are introduced in Table 1. It gives the WDS codes and names (discoverer designations) (Mason et al. 2001), the Hipparcos numbers and trigonometric parallaxes, combined visual magnitudes and spectral types. The remaining columns list the orbital parameters determined here (period $P$ and semimajor axis $a$ ), estimated component's masses, the angles $\Phi$ between the orbital momentum vectors, and the ratio of outer and inner periods $P_{2} / P_{1}$. The first system, LHS 1070, is composed of low-mass stars or brown dwarfs and has the smallest ratio of $P_{2} / P_{1}=4.5$, suggesting a 9:2 MMR. The members of the remaining three hierarchies have masses slightly below or above solar.

The method of orbit calculation by simultaneous fitting of the inner and outer pairs was presented by Tokovinin \& Latham (2017); it is briefly outlined in Section 2. Then in Section 3 the four hierarchies are described individually. A few similar systems discovered only recently are presented in Section 4 , the paper concludes by the short summary in Section 5 .

\section{CALCULATION OF TRIPLE-STAR ORBITS}

The objects for this study were selected among triple systems with one (or both) orbits already listed in the Sixth orbit catalog (Hartkopf et al. 2001). The available astrometry was extracted from the WDS database by B. Mason and complemented by recent speckle measurements at the Southern Astrophysical Research (SOAR) $4.1 \mathrm{~m}$ telescope, some of those still unpublished. One of the triples (HIP 9497) was discovered at SOAR in 2008, another (HIP 78842) was discovered the same year and with the same speckle instrument at the Blanco telescope (Tokovinin et al. 2010).

I use the IDL code orbit3.pro to fit simultaneously the position measurements of the inner and outer systems by two Keplerian orbits; see Tokovinin \& Latham (2017) for further information. The two orbits are defined by 20 orbital elements. However, as for the objects studied here the RVs are not available, only 15 "visual" elements are fitted. In this paper, the parameters of the inner orbit $(\mathrm{Ba}, \mathrm{Bb})$ are denoted by the index 1 , while the outer orbit $(\mathrm{A}, \mathrm{B})$ has the index 2 . The inner and outer orbits can be computed independently of each other, but then, to compute the outer orbit, the positions of the center of mass of the inner subsystem must be calculated using its orbit and the estimated component's masses. Simultaneous fitting avoids assumptions about masses and allows measurement of the inner mass ratio, as explained below.

Let $\mathrm{A}, \mathrm{B}$ be the outer pair and $\mathrm{Ba}, \mathrm{Bb}$ the inner subsystem belonging to the secondary component. The positions of $\mathrm{A}, \mathrm{Ba}$ reflect the motion in both orbits, resulting in the wavy trajectory (wobble). When the inner pair is not resolved, the measurements of $\mathrm{A}, \mathrm{B}$ refer to the photo-center of the subsystem $\mathrm{Ba}, \mathrm{Bb}$ and may still show a wobble of lower amplitude. The ratio of the wobble amplitude to the semimajor axis of the inner orbit, called here wobble factor, is $f=q_{1} /\left(1+q_{1}\right)$ for resolved measurements and $f^{*}=q_{1} /\left(1+q_{1}\right)-r_{1} /\left(1+r_{1}\right)$ for the photo-center measurements, where $q_{1}$ is the mass ratio in the inner pair and $r_{1}$ is the light ratio of its components. Let $\mathbf{x}_{\mathrm{A}, \mathrm{B}}$ be the position vector in the outer orbit, directed from $\mathrm{A}$ to the center of mass $\mathrm{B}$, and $\mathbf{x}_{\mathrm{Ba}, \mathrm{Bb}}$ the vector in the inner subsystem. Then the vector of the resolved position in the outer pair is

$$
\mathbf{x}_{\mathrm{A}, \mathrm{Ba}}=\mathbf{x}_{\mathrm{A}, \mathrm{B}}+f \mathbf{x}_{\mathrm{Ba}, \mathrm{Bb}} .
$$

The IDL code was modified here to treat both resolved and unresolved measurements of the outer pair (orbit3.pro allows only resolved measurements). If the inner subsystem is a twin with equal components, $f^{*}=0$, and unresolved measurements of the outer pair do not contain any wobble. When the subsystem belongs to the secondary component, the wobble factor is negative, but the relation $q_{1}=|f| /(1-|f|)$ still holds.

The angle $\Phi$ between the angular momentum vectors 
TABLE 2

Orbital Elements

\begin{tabular}{|c|c|c|c|c|c|c|c|c|}
\hline $\begin{array}{c}\text { WDS/system } \\
\text { Name }\end{array}$ & $\begin{array}{c}P \\
(\mathrm{yr})\end{array}$ & $\begin{array}{c}T \\
(\mathrm{yr})\end{array}$ & $e$ & $\begin{array}{c}a \\
\left({ }^{\prime \prime}\right)\end{array}$ & $\begin{array}{l}\Omega \\
\left({ }^{\circ}\right)\end{array}$ & $\begin{array}{l}\omega \\
\left({ }^{\circ}\right)\end{array}$ & $\begin{array}{c}i \\
\left({ }^{\circ}\right)\end{array}$ & $f$ \\
\hline 00247-2653/inner & 17.247 & 2006.440 & 0.0172 & 0.4598 & 14.82 & 202.53 & 62.04 & -0.485 \\
\hline LEI 1 BC & \pm 0.016 & \pm 0.007 & \pm 0.0008 & \pm 0.0007 & \pm 0.12 & fixed & \pm 0.11 & \pm 0.006 \\
\hline $00247-2653 /$ outer & 77.62 & 2049.67 & 0.039 & 1.528 & 13.9 & 210.7 & 62.5 & \\
\hline LEI $1 \mathrm{~A}, \mathrm{BC}$ & \pm 2.10 & \pm 1.32 & \pm 0.021 & \pm 0.112 & \pm 0.7 & \pm 6.4 & \pm 0.4 & \\
\hline 02022-2402/inner & 14.40 & 2006.400 & 0.058 & 0.102 & 120.5 & 227.2 & 152.5 & -0.473 \\
\hline TOK $41 \mathrm{Ba}, \mathrm{Bb}$ & \pm 0.89 & \pm 0.58 & \pm 0.051 & \pm 0.005 & \pm 13.2 & \pm 16.9 & \pm 4.3 & \pm 0.017 \\
\hline $02022-2402 /$ outer & 80.1 & 2041.75 & 0.336 & 0.414 & 179.5 & 0 & 180 & \\
\hline HDS 272 AB & \pm 6.1 & \pm 2.25 & \pm 0.043 & \pm 0.038 & \pm 3.3 & fixed & fixed & \\
\hline 05239-0052/inner & 47.03 & 1959.88 & 0.288 & 0.3366 & 321.03 & 277.7 & 87.60 & -0.592 \\
\hline A 847 B,C & \pm 0.36 & \pm 0.40 & \pm 0.014 & \pm 0.0013 & \pm 0.15 & \pm 0.80 & \pm 0.25 & \pm 0.016 \\
\hline 05239-0052/outer & 1200 & 2280 & 0.20 & 3.716 & 335.41 & 249.2 & 95.67 & \\
\hline WNC 2 A,BC & fixed & \pm 35 & fixed & \pm 0.020 & \pm 0.45 & \pm 7.8 & \pm 0.32 & \\
\hline 16057-3252/inner & 10.46 & 2012.623 & 0.240 & 0.1284 & 179.1 & 34.9 & 141.8 & -0.491 \\
\hline WSI 84 Ba,Bb & \pm 0.07 & \pm 0.027 & \pm 0.006 & \pm 0.0009 & \pm 1.4 & \pm 1.7 & \pm 0.8 & \pm 0.008 \\
\hline $16057-3252 /$ outer & 131.2 & 1981.4 & 0.029 & 0.807 & 161.7 & 53.3 & 152.3 & \\
\hline SEE 264 A,B & \pm 1.9 & \pm 4.0 & \pm 0.013 & \pm 0.070 & \pm 3.8 & \pm 12.6 & \pm 1.4 & \\
\hline
\end{tabular}

TABLE 3

RELATIVE POSITIONS AND RESIDUALS (FRAGMENT)

\begin{tabular}{|c|c|c|c|c|c|c|c|c|}
\hline WDS & Sys & $\begin{array}{c}\text { Date } \\
\text { (year) }\end{array}$ & $\begin{array}{c}\theta \\
\left(^{\circ}\right)\end{array}$ & $\begin{array}{c}\rho \\
\left({ }^{\prime \prime}\right)\end{array}$ & $\begin{array}{c}\sigma \\
\left({ }^{\prime \prime}\right)\end{array}$ & $\begin{array}{c}\mathrm{O}-\mathrm{C}_{\theta} \\
\left(^{\circ}\right)\end{array}$ & $\begin{array}{c}\mathrm{O}-\mathrm{C}_{\rho} \\
\left({ }^{\prime \prime}\right)\end{array}$ & $\operatorname{Ref}^{a}$ \\
\hline $02022-2402$ & $\mathrm{Ba}, \mathrm{Bb}$ & 2008.6990 & 187.9 & 0.0880 & 0.0050 & 0.1 & -0.0011 & $\mathrm{~s}$ \\
\hline $02022-2402$ & $\mathrm{Ba}, \mathrm{Bb}$ & 2008.7670 & 183.9 & 0.0830 & 0.0050 & -2.0 & -0.0065 & $\mathrm{~S}$ \\
\hline $02022-2402$ & $\mathrm{Ba}, \mathrm{Bb}$ & 2008.7670 & 186.5 & 0.0914 & 0.0050 & 0.6 & 0.0019 & $\mathrm{~S}$ \\
\hline $02022-2402$ & $\mathrm{Ba}, \mathrm{Bb}$ & 2009.6700 & 162.2 & 0.1018 & 0.0050 & 0.5 & 0.0060 & $\mathrm{~S}$ \\
\hline $02022-2402$ & $\mathrm{~A}, \mathrm{Ba}$ & 2008.7674 & 343.7 & 0.5814 & 0.0050 & -0.5 & 0.0008 & $\mathrm{~S}$ \\
\hline $02022-2402$ & $\mathrm{~A}, \mathrm{Ba}$ & 2009.6709 & 340.4 & 0.5851 & 0.0050 & -0.0 & 0.0016 & $\mathrm{~S}$ \\
\hline $02022-2402$ & $\mathrm{~A}, \mathrm{~B}$ & 1991.2500 & 28.0 & 0.5720 & 0.0100 & 3.0 & 0.0447 & $\mathrm{H}$ \\
\hline $02022-2402$ & $\mathrm{~A}, \mathrm{~B}$ & 2000.7670 & 0.6 & 0.5350 & 0.0050 & -1.1 & -0.0183 & $\mathrm{~s}$ \\
\hline
\end{tabular}

a References: G: Gaia; H: Hipparcos, K: Köhler et al. (2012); M: micrometer measures; P: photographic; S: speckle interferometry at SOAR, s: other speckle interferometry.

of the inner and outer orbits (mutual inclination) can be easily computed from the orbital elements $\Omega$ and $i$. However, without RV information the true ascending nodes of both orbits remain undefined; an alternative orbit with a pole reflected around the line of sight corresponds to the same on-sky measurements. Two alternative values of $\Phi$ result from this situation, and it is not known $a$ priori which angle is the correct one. A smaller angle is normally selected on statistical grounds, considering the orbit alignment tendency (Tokovinin 2017), and by using other arguments such as growth of the inner eccentricity by Kozai-Lidov cycles at large $\Phi$ (Naoz 2016). The last column of Table 1 gives both alternative angles $\Phi$ and the period ratio $P_{2} / P_{1}$.

The orbital elements derived here and their errors are listed in Table 2, in common notation. For the inner subsystems, the last column gives the wobble factor $f$. The individual position measurements and their residuals are provided in Table 3, available in full electronically. Its second column specifies the nature of the measurement: $\mathrm{Ba}, \mathrm{Bb}$ refers to the resolved inner pair, $\mathrm{A}, \mathrm{Ba}$ to the resolved outer pair, and A,B to the unresolved measure of the outer pair. As the errors of positional measures are either not provided in the original data sources or are unreliable, the errors in Table 3 are assigned subjectively, depending on the observing technique, to determine the relative weights. The errors of modern measures by adaptive optics and speckle interferometry at large telescopes are between 2 mas and 5 mas, the Hipparcos relative po- sitions are accurate to 10 mas, the photographic astrometry has errors of 30 mas, and the errors of visual micrometer measures range from 50 mas to 0.25 . Outlying visual measures are given artificially low weight to reduce their impact. The actual weighted rms residuals roughly match the adopted errors.

In the following Figures, I plot the outer trajectory (or its fragment) with the wobble included. One outer period is plotted, so the trajectory is not closed when the period ratio is not an integer number. Resolved measures are plotted as large asterisks and are connected to the ephemeris positions by short dotted lines. The unresolved measures of the outer pair are plotted by smaller symbols and also connected to their ephemeris positions corresponding to the smaller wobble factor $f^{*}$; these positions do not lie on the same trajectory. The inner orbit and measures are over-plotted by the dashed line and triangles around the same center, even though here they depict the motion of the secondary subsystem. In all plots the scale is in arcseconds, North is up, East is left.

\section{INDIVIDUAL SYSTEMS}

The four hierarchical systems featured in Table 1 are discussed in the following sub-sections.

\subsection{7-2653 (LHS 1070)}

The triple system LHS 1070, also known as GJ 2005, LP 881-64, and WDS J00247-2653, is located at $7.7 \mathrm{pc}$ from the Sun. The recent parallax of $132.3 \pm 11.4$ mas 


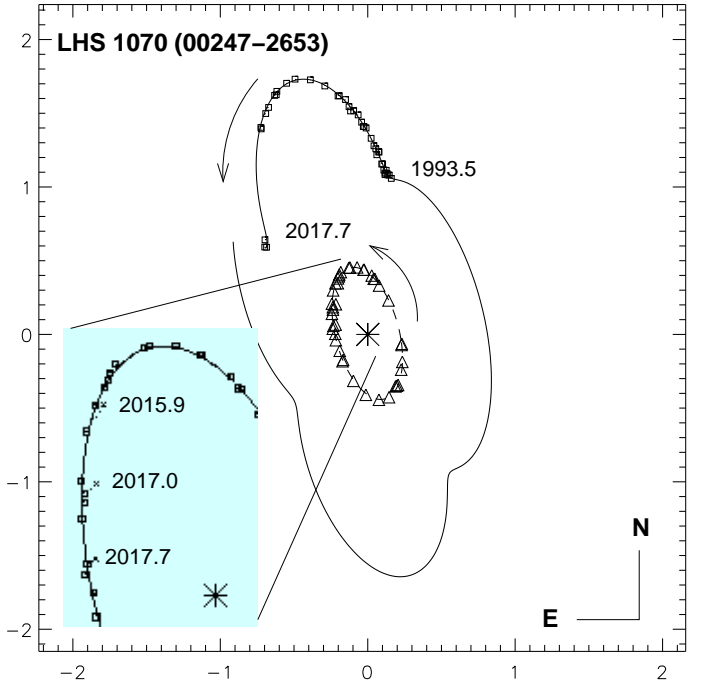

FIG. 2. - The orbits of LHS 1070 (WDS J00247-2653). In this and subsequent plots, the axis scale is in arcseconds. The insert shows fragment of the inner orbit where the deviating measures made in 2015-2017 are plotted by crosses.

given by Weinberger et al. (2016) is less accurate than $129.5 \pm 2.5$ mas measured by Costa et al. (2005), so the latter value is used here. The triple system consists of two stars $\mathrm{B}$ and $\mathrm{C}$ with masses around $0.07 \mathcal{M}_{\odot}$ (near the hydrogen burning limit) in a 17.25 year orbit around each other. They are accompanied by the more distant and massive primary component A. Detailed analysis of this hierarchy, including the review of relevant literature, was presented by Köhler et al. (2012). These authors have shown that the system is dynamically stable only when the outer period exceeds $\sim 80$ years and the outer eccentricity is small (their Fig. 5). Yet, they preferred the unstable outer orbit of 44 year period that provided the best fit to the data available at the time, covering the period from 1993 to 2008. This orbit still figures in the catalog (Hartkopf et al. 2001), challenging common dynamical stability criteria. New measurements made at SOAR in 2015-2017 lead to a different outer orbit and resolve the discrepancy.

The revised orbital elements and their errors are listed in Table 2, I have chosen to fix the inner angle $\omega_{1}$ in the final fit to avoid a large error of $T_{1}$ (the inner eccentricity is small and $\omega_{1}$ strongly correlates with $\left.T_{1}\right)$. The wobble factor $f=0.485 \pm 0.006$ means $q_{1}=f /(1-f)=0.942$.

The inner orbit computed here is essentially identical to that by Köhler et al. (2012), as it already has been defined with a high precision by prior measurements. The parallax of $129.5 \pm 2.5$ mas (Costa et al. 2005) leads to the inner mass sum of $0.150 \pm 0.009 \mathcal{M}_{\odot}$, hence the individual masses of 0.077 and $0.070 \mathcal{M}_{\odot}$. Note, however, that the latest measures of B,C at SOAR have large and similar residuals to the new orbit $\left(-6^{\circ}\right)$ that cannot be removed by its adjustment. This is most likely caused by the real deviations of the inner subsystem from the Keplerian motion owing to its interaction with the tertiary. A full dynamical analysis of this system is in order. Meanwhile, the SOAR measures of $\mathrm{B}, \mathrm{C}$ were given reduced weight in the orbit fit.

The outer orbit converged to a solution that is almost exactly coplanar with the inner orbit. The period ratio is

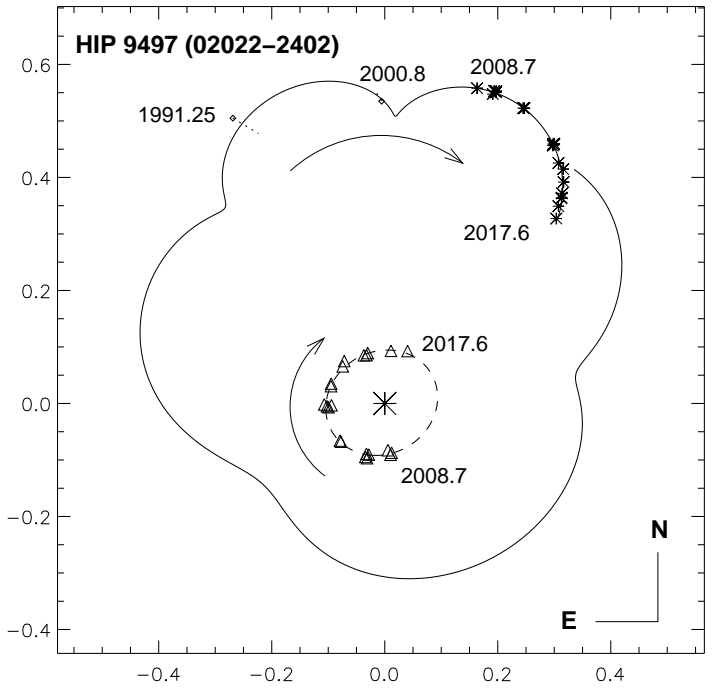

FIG. 3.- The orbits of HIP 9497 (WDS J02022-2402, HDS 272 $\mathrm{A}, \mathrm{B}$ and TOK $41 \mathrm{Ba}, \mathrm{Bb})$.

$4.50 \pm 0.03$. Note that the inner and outer lines of apsides are aligned (similar $\omega$ ). Figure 2 shows both orbits. The outer mass sum of $0.273 \mathcal{M}_{\odot}$ leads, by subtraction, to the mass of $\mathrm{A}, 0.123 \mathcal{M}_{\odot}$.

The period ratio of 4.5 is slightly less than the 4.7 canonical stability limit derived by Mardling \& Aarseth (2001) for coplanar triples. The system is thus on the verge of dynamical stability, implying strong dynamical interaction between the orbits. It is very likely that this interaction drives the periods into a MMR. The period ratio found here implies the 9:2 MMR.

The orbit coplanarity strongly suggests viscous evolution in a disk as the formation mechanism of this triple system. The pair B,C formed first and migrated to the present-day separation of 3.5 au as a result of accretion, until the outer companion A overtook the accretion flow. The orbit of A also evolved to a smaller separation, reaching its present axis of $12 \mathrm{au}$. If the migration proceeded further, the triple system would have broken up into a binary and a single star. The fact that migration stopped just before the break-up explains the rarity of similar quasi-stable triple systems.

Köhler et al. (2012) noted that the component A is $\sim 3 \mathrm{mag}$ too faint for its mass (or over-massive for its luminosity). They suggested that A could be itself a close binary, but have refuted its resolution into a closer pair, reported previously. The new outer orbit leads to a slightly smaller mass of A, but does not resolve the mass discrepancy.

\subsection{2-2402 (HIP 9497)}

The triple system HIP 9497 was discovered in 2008 nearly simultaneously and independently at SOAR (Tokovinin et al. 2010) and WIYN (Horch et al. 2012) telescopes by resolving the faint secondary component of the Hipparcos binary HDS 272 into a close pair TOK 41 $\mathrm{Ba}, \mathrm{Bb}$. Now about $60 \%$ of the inner orbit is covered and its elements are well constrained. However, in 26 years elapsed since the discovery of the outer pair A,B, only a third of its orbit is covered, so its elements are still uncertain. Here the previous tentative 138 year orbit of 


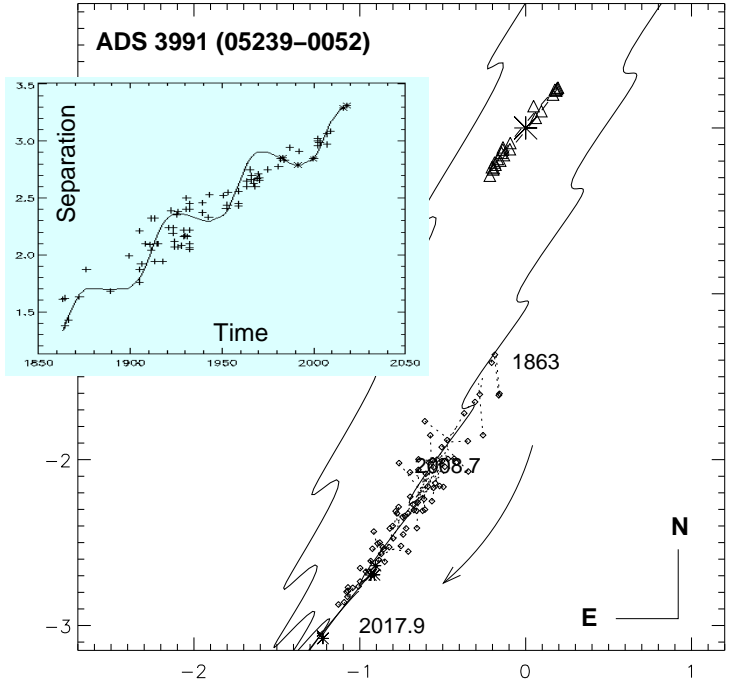

FIG. 4.- Orbits of ADS 3991 (WDS J05239-0052). The insert shows the separation between A and BC (plus signs) and A and B (asterisks) vs. time, the wavy line is the modeled separation of $\mathrm{A}$ and B.

A,B is updated to $P_{2}=80$ years. I reprocessed the published SOAR measure of 2008.76 where $\mathrm{Ba}$ and $\mathrm{Bb}$ were swapped. As the outer orbit is seen almost face-on, I fixed its inclination to $i_{2}=180^{\circ}$, which makes the two angles $\Omega_{2}$ and $\omega_{2}$ degenerate; hence, $\omega_{2}=0$ is also fixed. The period ratio is obviously small, $P_{2} / P_{1}=5.6 \pm 0.7$. The still low accuracy of the period ratio does not allow to make any statements on the MMR, although the resonance is likely. The resolved measurements of $\mathrm{A}, \mathrm{Ba}$ clearly show the wavy motion caused by the inner orbit (Figure 3).

The Hipparcos parallax of $16.09 \pm 0.76$ mas is adopted here because the Gaia (Gaia Collaboration 2016) parallax of $17.31 \pm 0.99$ mas is less accurate. The parallax and the inner orbit correspond to the inner mass sum of $1.22 \pm 0.17 \mathcal{M}_{\odot}$, hence the masses of 0.64 and 0.58 for $\mathrm{Ba}$ and $\mathrm{Bb}$, respectively (the wobble factor $f=-0.473 \pm 0.020$ implies the inner mass ratio of 0.90 ). They agree with the masses estimated from the absolute magnitudes (the interstellar extinction is negligible for these nearby systems). The less well defined outer orbit gives the $1.44 \mathcal{M}_{\odot}$ mass of $\mathrm{A}$, matching its spectral type F6V. This triple system, like the previous one, is a double twin.

The inner orbit is almost circular $\left(e_{1}=0.06 \pm 0.05\right)$, while the outer eccentricity $e_{2}=0.33 \pm 0.04$ is moderate. As one of the orbits has a face-on orientation, the two alternative inclination angles coincide, $\Phi=27^{\circ}$. At such mutual inclination, there are no Kozai-Lidov cycles.

\subsection{9-0052 (HIP 25240)}

This is a bright $2+2$ quadruple system HR 1782 (ADS 3991, HIP 25240). The double-lined spectroscopic pair Aa,Ab has a period of 22.58 (Tokovinin 1997), while the visual pair B,C has a well-defined visual orbit with $P_{1}=$ 47 years. The two inner pairs revolve around each other on a wide, poorly constrained outer orbit with $P_{2} \sim 1000$ years. Given this freedom, I chose to fix the outer period $P_{2}=1200$ years and the outer eccentricity $e_{2}=0.2$ in the combined orbit fit, which gives then a reasonable outer mass sum. The published measure of the $3^{\prime \prime} \mathrm{A}, \mathrm{B}$ pair made at SOAR in 2015.9 was distorted by aliasing; here it is corrected and confirmed by the fresh measure in 2017.9. The last unresolved measure of A,BC is provided by Gaia.

Both inner and outer orbits are highly inclined and nearly parallel on the sky (Figure 41). The wobble is therefore parallel to the outer trajectory and affects mostly the separation. Indeed, the five resolved measures of A,B made from 1983 to 2017 by speckle interferometry and Hipparcos demonstrate the 47-year wave in the separation and define the wobble factor $f=-0.592 \pm 0.016$. This means that the component $\mathrm{C}$ is more massive than $\mathrm{B}, q_{\mathrm{B}, \mathrm{C}}=1.44$.

The Hipparcos parallax of $17.95 \pm 0.77$ mas could be biased by the complex nature of this source consisting of three resolved stars. The Gaia parallax of $18.75 \pm 0.47$ mas results in the inner mass sum of $\mathrm{B}, \mathrm{C}$ of $2.62 \mathcal{M}_{\odot}$, larger than $2.3 \mathcal{M}_{\odot}$ derived from the absolute magnitudes in (Tokovinin 1997). Considering the large wobble amplitude, it is likely that the subsystem B,C actually contains an additional close companion to $\mathrm{C}$ with a mass of $\sim 0.5 \mathcal{M}_{\odot}$; the derived masses of $\mathrm{B}$ and $\mathrm{C}$ are 1.07 and $1.55 \mathcal{M}_{\odot}$, respectively. The mass sum in the outer orbit is $5.4 \mathcal{M}_{\odot}$.

The RVs of A (center of mass of Aa,Ab) and BC, measured around 1994 by Tokovinin (1997), were 55.0 \pm 0.2 and $52.1 \pm 0.2 \mathrm{~km} \mathrm{~s}^{-1}$, respectively. The RV difference between $\mathrm{A}$ and $\mathrm{BC}$ could be used to further constrain the outer orbit by requiring that the RV amplitudes match the expected mass sum. This can be achieved by imposing the constraint $\omega_{2}=300^{\circ}$, leading to a shorter $P_{2}$ and a larger $e_{2}$. However, the measured RV of BC (combined light of both components) could be biased by the orbital motion in this pair, so I do not trust its value and only assume that the sign of the difference is correct and thus defines the true ascending node of the outer orbit.

Although the outer orbit is poorly constrained, its nearly edge-on orientation allows a robust estimate of the relative inclination between the orbits of $\mathrm{A}, \mathrm{BC}$ and $\mathrm{B}, \mathrm{C}: \Phi=16.5$. As the ascending node of $\mathrm{B}, \mathrm{C}$ is not known, the alternative angle $\Phi=165^{\circ}$ is also possible, although less likely.

\subsection{7-3252 (HIP 78842)}

The hierarchical system HIP 78842 (Hipparcos parallax $24.70 \pm 1.96$ mas) is fortunate in the coverage of both its orbits. The outer pair SEE 264 A,B, discovered in 1897, has made almost one full revolution, defining its 131 year nearly circular face-on orbit quite well. The secondary component B was resolved into a close pair WSI $84 \mathrm{Ba}, \mathrm{Bb}$ in 2008.55 (Tokovinin et al. 2010) and has also completed almost one full revolution; its 10 year orbit is computed here for the first time. The rms residuals of the 11 speckle measurements of $\mathrm{Ba}, \mathrm{Bb}$ to this orbit are remarkably small, only 1.4 mas.

Figure 5 illustrates the "dancing" orbital motion in this triple system. The two orbits are nearly coplanar, $\Phi=14^{\circ}$ (the alternative inclination $\Phi=65^{\circ}$ would cause Kozai-Lidov cycles and hence is unlikely). The period ratio of $12.6 \pm 0.2$ is small, while the MMR cannot be ruled out at the current accuracy. The wobble factor $f=$ $-0.491 \pm 0.008$ leads to $q_{1}=0.96$, confirming that the inner subsystem $\mathrm{Ba}, \mathrm{Bb}$ is a twin. The unresolved historic 


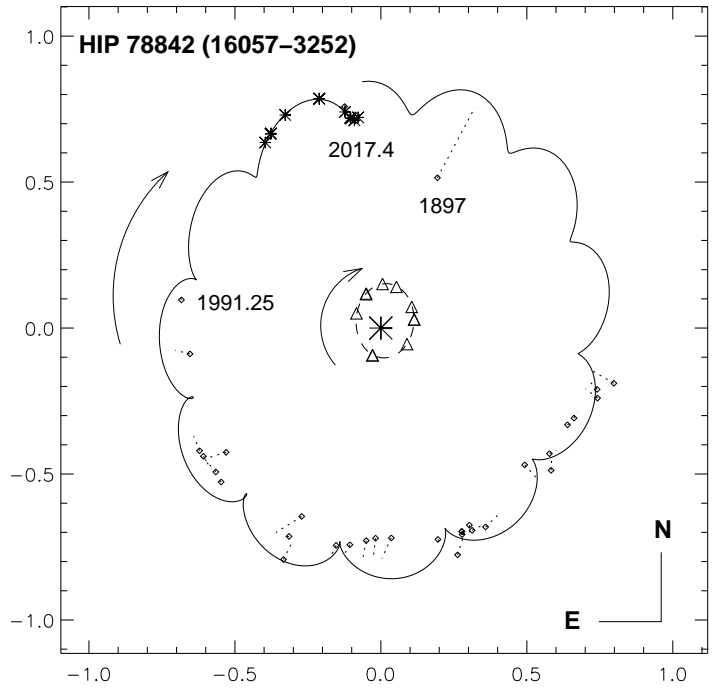

FIG. 5.- The orbits of HIP 78842 (WDS J16057-3252, SEE $264 \mathrm{~A}, \mathrm{~B}$ and WSI $84 \mathrm{Ba}, \mathrm{Bb})$.

measurements of A,B do not show any wobble, and this is taken into account in the orbital fit (for this reason, most short dotted lines in Figure 5 do not touch the outer trajectory depicted by the full line). The measure by Mason et al. (2011) was corrected because $\mathrm{Ba}$ and $\mathrm{Bb}$ were swapped and the outer measure refers to $\mathrm{A}, \mathrm{Bb}$, not to A,B as published. The last unresolved measure of A,B is furnished by Gaia.

Considering the large error of the Hipparcos parallax, I prefer to use the dynamical parallax of 24.6 mas derived from the inner orbit by adopting the masses of 0.75 $\mathcal{M}_{\odot}$ for $\mathrm{Ba}$ and $\mathrm{Bb}$, estimated from their absolute magnitudes. The mass of A derived in the same way is 0.96 $\mathcal{M}_{\odot}$. The less accurate outer orbit gives the matching dynamical parallax of 23.3 mas.

Unlike other hierarchies studied here, HIP 78842 is not a double twin. It is actually a $3+1$ quadruple, considering the companion $\mathrm{C}$ at $9^{\prime \prime} \cdot 3$ separation with matching proper motion, RV, and photometric distance; the estimated period of $\mathrm{AB}, \mathrm{C}$ is $\sim 4 \mathrm{kyr}$. The estimated mass of $\mathrm{C}, 0.67 \mathcal{M}_{\odot}$, is similar to the masses of $\mathrm{Ba}$ and $\mathrm{Bb}$; its spectral type is $\mathrm{K} 5 \mathrm{~V}$. The combined color of $\mathrm{AB}$ is bluer than that of $\mathrm{C}$ : their $V-K$ indices are $2.46 \mathrm{mag}$ and $3.32 \mathrm{mag}$, respectively.

The quasi-circular and aligned orbits of $\mathrm{A}, \mathrm{B}$ and $\mathrm{Ba}, \mathrm{Bb}$ strongly suggest that this hierarchy was formed in a rotating disk, as discussed in Section 1. Moreover, the circularity of the A,B orbit implies the absence of KozaiLidov cycled caused by the outer companion C, so its orbit should also be inclined by $<39^{\circ}$ relative to the orbit of A,B. The presence of $\mathrm{C}$ fits the sequential formation scenario and possibly explains why the component A could not grow to become a double twin: the gas supply coming to A was re-directed to the distant component $\mathrm{C}$ when it formed. Something similar may have happened in the "planetary" $3+1$ quadruple system HD 91962 (Tokovinin et al. 2015), where all three secondary components are less massive than the primary star.

\section{RECENTLY DISCOVERED DOUBLE TWINS}
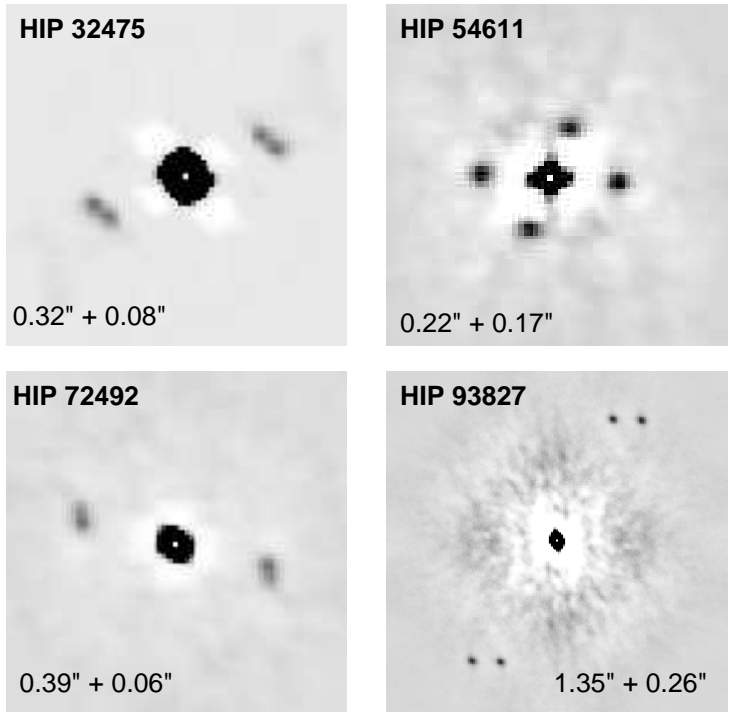

Fig. 6.- Double twins discovered at SOAR in 2015 and 2016. Each panel shows the speckle auto-correlation function in arbitrary scale and in negative intensity stretch. The Hipparcos numbers and separations are indicated.

TABLE 4

DOUBLE TWINS DISCOVERED AT SOAR

\begin{tabular}{ccccc}
\hline \hline WDS & HIP & $\begin{array}{c}M_{1} \\
\left(\mathcal{M}_{\odot}\right)\end{array}$ & $\begin{array}{c}P_{\text {out }}^{*} \\
(\mathrm{yr})\end{array}$ & $\begin{array}{c}P_{\text {in }}^{*} \\
(\mathrm{yr})\end{array}$ \\
\hline $06467+0822$ & 32475 & 1.45 & 64 & 11 \\
$11106-3234$ & 54611 & 1.96 & 900 & 113 \\
$14494-5726$ & 72492 & 1.59 & 190 & 19 \\
$19064-1154$ & 93827 & 1.11 & 200 & 31 \\
\hline
\end{tabular}

The luminosity of dwarf stars is a strong function of their mass. In a double twin triple system, the combined light of $\mathrm{Ba}$ and $\mathrm{Bb}$ is much fainter than the light of $\mathrm{A}$. Binaries with a large magnitude difference $\Delta m$ are difficult to discover. Consequently, the number of double twins among low-mass stars in the solar neighborhood could be substantially larger than known currently.

New observing techniques such as adaptive optics and speckle interferometry at large telescopes help to discover double twins by resolving secondary components of known binaries with a large $\Delta m$ into inner pairs. Four such discoveries made at SOAR in 2015 (Tokovinin et al. 2016) and 2016 (Tokovinin et al. 2018) are shown in Table 4 and illustrated in Figure [6. All are previously known binaries with primary components slightly more massive than the Sun, where the faint secondary components have been resolved at SOAR into close pairs composed of equal stars. The orbital periods $P^{*}$, estimated crudelyfrom the separations, indicate that after several decades of monitoring the inner and outer orbits will become defined and the architecture of these hierarchies will become known. The system HIP 54611 looks non-hierarchical. However, the outer pair was discovered in 1879 at a larger $1^{\prime \prime} 0$ separation and has closed down since; apparently, the inner subsystem B,C now projects on the primary component A.

The latest version of the Multiple Star Catalog, MSC (Tokovinin 2018) contains many other double twins and $2+2$ quadruples; their orbital periods range from days to kilo-years. Some of those hierarchies are composed 


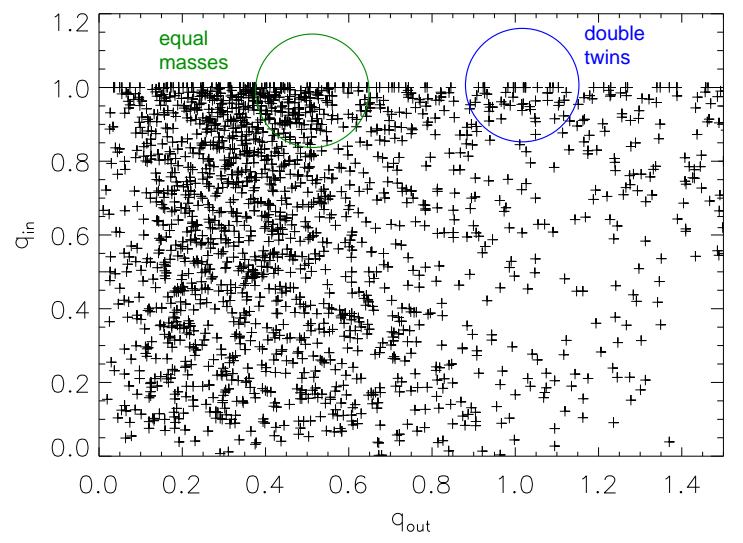

FIG. 7.- Comparison of mass ratios at adjacent hierarchical levels for multiple systems from the MSC with primary mass $<1.5$ $\mathcal{M} \odot$.

of low-mass stars. To give just two examples, WDS J04185+2817 consists of two resolved pairs of M3.5V dwarfs, DD Tau and SZ Tau, separated by $30^{\prime \prime}$ (estimated outer period $3.8 \mathrm{kyr}$ ). The WDS J05101-2341 is also a $2+2$ quadruple of similar architecture with M3V components.

\section{SUMMARY AND DISCUSSION}

The four hierarchical systems studied here have several features in common: moderate ratios of inner and outer periods, approximately co-aligned orbits with small eccentricity, and similar masses in the inner and outer subsystems (double twins). As outlined in Section 1, such hierarchies could be produced by sequential formation and migration of their components at the epoch of mass assembly, i.e. at the protostellar phase. It is difficult to imagine such well-organized systems to be products of chaotic dynamics.

Sequential formation may not always produce double twins. If the outer companion forms by the end of the accretion phase, it may not grow in mass sufficiently to equal the inner pair. Alternatively, the growth of the outer companion can be stopped by formation of another, more distant star, as might have happened in the case of HIP 78842 .

The triple system LHS 1070 is remarkable by its low total mass of $0.27 \mathcal{M}_{\odot}$. However, it does not hold the record in this respect. The two lowest-mass triples in the MSC, WDS J02055-1159 and J08382+1511, have the estimated total masses of 0.150 and $0.165 \mathcal{M}_{\odot}$, respectively, and are composed of brown dwarfs. Their architecture is similar to that of LHS 1070: they consist of the primary component A and the secondary subsystem $\mathrm{B}, \mathrm{C}$ with a moderate ratio of separations and the estimated periods of a few decades (the orbits are not known yet). However, neither of these sub-stellar hierarchies are double twins because the mass of $\mathrm{A}$ is less than the total mass of the inner subsystem.

Figure 7 compares the mass ratios at two adjacent hierarchical levels computed for all systems from the MSC with primary mass less than $1.5 \mathcal{M}_{\odot}$. As this catalog is burdened by large selection effects, the Figure does not represent unbiased statistics. However, if the mass-ratio distribution has sharp details, they could probably be seen in this plot because observational selection is expected to be a smooth function of parameters. Indeed, concentration of the inner mass ratios toward one (twins) is evident. On the other hand, the points do not show any clustering at the $(1,1)$ location corresponding to double twins. The density of points is larger near $(0.5,1)$, i.e. when all three components have comparable masses. However, as noted above, the discovery of double twins may have been seriously hampered until now.

Determination of inner and outer orbits in triple systems is paced by the accumulation of measurements. In most cases, the available time coverage does not yet allow meaningful analysis of long outer orbits. The work presented here can be expanded to include several other hierarchies, but their number will remain modest and will grow only slowly with time. On the other hand, discovery of double twins using modern high-resolution techniques is a promising undertaking, especially when applied to nearby low-mass stars. Systematic surveys of such volume-limited samples with high angular resolution are under way already (e.g. Law et al. 2010).

Some data used here were obtained at the Southern Astrophysical Research (SOAR) telescope. This work used the SIMBAD service operated by Centre des Données Stellaires (Strasbourg, France), bibliographic references from the Astrophysics Data System maintained by SAO/NASA, and the Washington Double Star Catalog maintained at USNO. I thank B. Mason for extracting historic measurements from the WDS database.

\section{REFERENCES}

Artymowicz P., Lubow S. H., 2001, in "The Formation of Binary Stars", Proc. IAU Symp. 200, eds. H. Zinnecker \& R. D. Mathieu (ASP: San Francisco), p. 439.

Costa, E., Méndez, R. A., Jao, W.-C., et al. 2005, AJ, 130, 337

Fabrycky, D. C., Lissauer, J. J., Ragozzine, D. et al. 2014, ApJ, 790,146

Gaia Collaboration, Brown, A. G. A., Vallenari, A., Prusti, T. et al. 2016, A\&A, 595, 2

Guszejnov, D., Hopkins, P. F., \& Krumholz, M. R. 2017 , MNRAS, 468, 4093

Hartkopf, W. I., Mason, B. D. \& Worley, C. E. 2001, AJ, 122, 3472

Heintz, W. D. 1996, AJ, 111, 408

Horch, E. P., Bahi, L. A. P., Gaulin, J. R. et al. 2012, AJ, 143, 10

Köhler, R., Ratzka, T. \& Leinert, Ch. 2012, A\&A, 541, 29

Law, N. M., Dhital, S., Kraus, A. et al. 2010, ApJ, 720, 1727

Lee, M. H., Fabrycky, D. \& Lin, D. N. C. 2013, ApJ, 774, 52
Mardling, R. A. \& Aarseth, S. J. 2001, MNRAS, 321, 398

Mason, B. D., Wycoff, G. L., Hartkopf, W. I., Douglass, G. G. \& Worley, C. E. 2001, AJ, 122, 3466 (WDS)

Mason, B. D., Hartkopf, W. I., Raghavan, D. et al. 2011, AJ 142, 176

Naoz, S. 2016, ARAA, 54, 441

Norman, M. L. \& Wilson, J. R. 1978, ApJ, 224, 497

Tobin, J. J., Kratter, K. M., Persson, M. V. et al. 2016, Nature, 538,483

Tokovinin, A. A. 1997, A\&AS, 121, 71

Tokovinin, A. 2008, MNRAS, 389, 925

Tokovinin A., Mason B. D., \& Hartkopf W. I. 2010, AJ, 139, 743

Tokovinin, A., Latham, D. W. \& Mason, B. D. 2015, AJ, 149, 195

Tokovinin, A., Mason, B. D., Hartkopf, W. I. et al. 2016, AJ, 151, 153

Tokovinin, A. \& Latham, D. W. 2017, ApJ, 838, 54 
Tokovinin, A., Mason, B. D., Hartkopf, W. I. et al. 2018, AJ, submitted

Tokovinin, A. 2017, ApJ, 844, 103

Tokovinin, A. 2018, ApJS, accepted (preprint arXiv:1712.04750

Facility: SOAR
Weinberger, A. J., Boss, A. P., Keiser, S. A. et al. 2016, AJ, 152, 24

Xu, X.-B., Xia, F., \& Fu, Y.-N. 2015, RAA, 15, 1857 